\title{
Une mort
}

\section{ou des morts?}

\section{Philippe Charlier ${ }^{1-3}$}

Depuis 2012, l'Organisation mondiale de la santé (OMS) définit la mort comme «la disparition permanente et irréversible de la capacité de conscience et de toutes les fonctions du tronc cérébral ».

La mort clinique correspond à un arrêt cardio-respiratoire prolongé empêchant l'oxygénation de l'organisme. Jusque dans les années 1950, tout arrêt cardiaque signifiait le décès. Mais l'invention du respirateur artificiel en 1952, par le médecin anesthésiste danois Björn Ibsen, a révolutionné cette définition de la mort, en permettant la mise en place des soins de réanimation et de soins intensifs. Quelques années plus tard, en 1959, le neurologue Maurice Goulon et l'infectiologue Pierre Mollaret de l'hôpital Claude-Bernard à Paris, proposent le concept de «coma dépassé », correspondant à l'état de mort encéphalique ${ }^{1}$. Ce concept sera repris dans le monde anglo-saxon en 1968, sous l'impulsion du comité de la Harvard Medical School (HMS), la faculté de médecine de l'université Harvard, à Boston, États-Unis [1]. Cette «mort cérébrale » correspond à l'absence irréversible d'activité cérébrale constatée par deux électro-encéphalogrammes (દદG) plats et aréactifs réalisés sur une durée d'au moins trente minutes chacun et espacés d'au moins quatre heures, ou d'une artériographie/angiographie par tomodensitométrie (TDM) objectivant une absence de flux sanguin intra-crânien. La mort peut donc être déclarée chez un sujet ayant une activité cardiaque persistante. Plus récemment, les pratiques de prélèvement et dons d'organe ont abouti à une modification de la définition de la mort clinique, qui peut être constatée après cinq minutes d'arrêt cardiaque chez des patients faisant l'objet d'un accompagnement dans le cadre d'une procédure d'arrêt des soins (limitation thérapeutique).

Deux éléments conduisent toutefois à relativiser ces définitions actuelles de la mort en Occident: d'abord, les récentes avancées technologiques tendant à montrer le caractère multi-focal, hétérogène, irrégulier et progressif de l'installation de la mort biologique. Ensuite, l'implication de plus en plus forte des sciences

Vignette (Photo (C) Inserm - Guimont Damien).

${ }^{1}$ Mollaret P, Goulon M. Le coma dépassé. Revue Neurologique $1959 ; 101$ (1) : 3-15. Présenté à la Journée de réanimation de l'hôpital Claude-Bernard, le 7 octobre 1959.

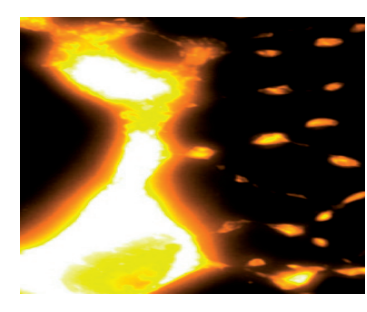

${ }^{1}$ Département de la recherche et de l'enseignement, musée du quai Branly-Jacques Chirac, 222 rue de l'Université, 75007 Paris, France.

${ }^{2}$ Laboratoire anthropologie, archéologie, biologie (LAAB), université Paris-Saclay (UVSQ), UFR des sciences de la santé, 2 avenue de la source de la Bièvre, 78180 Montigny-Le-Bretonneux,

humaines (anthropologie, sociologie, ethnologie) dans le champ disciplinaire des sciences fondamentales, permettant «d'enrichir le discours », et d'introduire une relativisation des points de vue vis-à-vis de l'arrêt France.

${ }^{3}$ Fondation anthropologie, archéologie, biologie (FAAB) Institut de France, 23 quai de Conti, 75007 Paris, France. philippe.charlier@uvsq.fr complet des fonctions vitales.

Une nouvelle notion a également émergé depuis quelques années, celle de la «mort sociale », qu'il faut aussi intégrer à cette remise en question du caractère monolithique de la mort: en mort sociale, les individus sont encore physiquement viables (parfois physiologiquement amoindris), mais leur isolement et/ou leur invisibilité les plongent dans une disparition complète vis-à-vis de la communauté des vivants [2]. Nous laisserons cependant de côté cette dernière notion (anthropologique), et nous nous concentrerons ici sur les diverses définitions biologiques de la mort, et leur grande relativité.

\section{Non immédiateté de la mort}

Les récents travaux de Dreier et al., Charité-Universitätsmedizin à Berlin, Allemagne, ont montré que la mort ne survient pas en un coup brutal: entre le moment de l'arrêt cardiaque définitif et l'arrêt total de l'activité cérébrale, environ dix minutes s'écoulent, marquées par un «tsunami électrique cérébral » [3]. Plutôt que l'extinction progressive de l'activité neuronale qu'on imaginait, c'est plus d'une vague électrique dont il s'agit, sorte de dernier feu d'artifice obéissant à une chronologie stéréotypée chez les patients observés en train de mourir (Tableau I).

Peut-on dire pour autant qu'un électro-encéphalogramme plat ne signifie pas la mort de l'individu, puisqu'on ne sait pas encore si l'on se situe avant ou après cette «vague de dépolarisation terminale »? Il a été proposé que les expériences de mort imminente (EMI) puissent correspondre à de telles dépolarisations (partielles plutôt que totales, car leur caractère total irait dans le sens de leur irréversibilité) de 


\begin{tabular}{ll}
\hline 0 seconde & Arrêt cardio-respiratoire \\
\hline $20-30$ secondes & Perte de connaissance (activité électrique ralentie) \\
\hline 30 secondes à $2-5$ minutes & Neurones « en veille (activité électrique quasi-inexistante) \\
& $\begin{array}{l}\text { Vague de dépolarisation terminale (irréversible : mort neuronale). } \\
\text { Son point de départ anatomique varie selon les patients. Chez les rongeurs, il semble s'agir } \\
\text { systématiquement du cortex somato-sensoriel. }\end{array}$ \\
\hline 10 minutes & Décès réel \\
\hline
\end{tabular}

Tableau I. Chronologie de l'activité électrique neuronale au cours d'un arrêt cardio-respiratoire prolongé.

zones particulièrement sensibles à l'hypoxie, en raison de leur forte densité neuronale: lobe temporal (hippocampe, principalement) et carrefour temporo-pariétal [4]. Une autre hypothèse tiendrait à une libération terminale (alors que le décès n'est pas encore irréversible, donc au cours de cette pseudo-agonie cérébrale) de substances de type hallucinogène [5].

Dans le même sens que cet ultime sursaut neuronal, il faut rapporter ces épisodes de «lucidité terminale » correspondant à de courts moments (10 minutes environ), uniques, survenant dans les huit jours précédant le décès, de récupération quasi-complète des fonctions cognitives, chez des patients déments ou touchés par des maladies neuro-dégénératives..., ce qui peut faire douter du caractère définitif de telles altérations de la conscience et des fonctions cognitives [6].

\section{L'aréactivité n'est pas la mort}

Les progrès de l'imagerie fonctionnelle cérébrale (neuro-imagerie) amènent à des modifications récentes de l'échelle de diagnostic des états de conscience altérée (passage de l'échelle CRS-R [revised coma recovery scale] à celle de SECONDS [simplified evaluation of consciousness disorders]). Les quatre nouveaux stades de conscience altérée désormais acceptés par la communauté scientifique sont donc: le syndrome locked-in (état d'éveil et de conscience normaux, mais le corps est presque entièrement paralysé en raison d'un accident vasculaire du tronc cérébral) ; l'état d'éveil répondant (sujet éveillé, yeux ouverts, signes clairs mais limités de conscience); l'état d'éveil non répondant (sujet éveillé, yeux ouverts, sans signe de conscience de soi ni de son environnement); et le coma (sujet non éveillé, yeux fermés, même lors de stimulation, sans signe de conscience) [7].

D'autres travaux plus récents montrent bien qu'une inactivité clinique chez un patient en coma profond ne correspond pas pour autant à une absence d'activité neuronale adaptée: ainsi, en réanimation, près de $15 \%$ des patients inconscients présentent en réalité des signes électriques de réponse cérébrale à des instructions motrices [8]. Si cette étude démontre qu'il n'y a pas présence ou absence de conscience mais qu'il existe au contraire une sorte de zone frontière, « d'entre deux », un même modèle n'est-il pas applicable à la mort? Cette interrogation doit cependant être pondérée, car tout dépend en réalité de la cause du coma et de la perte de conscience du sujet (contexte post-traumatique, de post-accident vasculaire cérébral [AVC] et de situations post-anoxoischémiques, etc.), dont les conséquences et le pronostic peuvent être totalement différents et variés.

\section{La théorie de la relativité appliquée à la mort}

Après l'arrêt du cœur, des trémulations musculaires peuvent persister, des battements cardiaques isolés également. Ces mouvements, ou sensations, sont particulièrement perturbants pour les familles qui peuvent alors douter de la réalité de la mort. Et pour cause, c'est que la mort s'installe et, pour s'installer, elle prend son temps.

L'hypothermie relativise encore plus cette chronologie - elle-même relative - de l'installation de la mort, avec des cas rapportés de réanimation efficace sans séquelle neurologique jusqu'à près de 9 heures d'état de mort apparente [9].

La mort ne signifie pas, sur le plan moléculaire, un arrêt global et total des activités enzymatiques et glycoprotéiques: plusieurs travaux ont bien montré une poursuite (voire même une intensification) de l'activité de transcription génique en post-mortem, puis une stagnation, et un arrêt définitif après un délai pouvant aller de 24 heures (chez l'homme) jusqu'à quatre jours chez certaines espèces [10-12].

Quel est le moment de la mort, alors? Celui du début de cette dépolarisation et/ou hyper-transcription génique? Actuellement oui. Mais c'est vraisemblablement une erreur, car cette vague électrique et/ou moléculaire est temporairement réversible (en cas de restauration d'une circulation sanguine, par exemple). Est-ce celui du moment irréversible de la dépolarisation, un peu plus tard? Celui de la fin de la dépolarisation, lorsque tout semble fini (jusqu'à preuve du contraire... car on a bien compris que ces notions sont particulièrement relatives)? 


\section{II n'y a pas de mort brutale : il n'y a que des morts lentes}

Les religions extrême-orientales nous enseignent que la mort n'est pas immédiate, entière, totale, mais au contraire une succession de lumières qui s'éteignent « le temps que dure un repas », pour reprendre la belle expression du Bardö Thodol (le Livre des morts tibétain) ${ }^{2}$. Cette caractéristique correspond bien aux dernières données moléculaires et d'exploration fonctionnelle issues de la recherche médicale [13].

Que nous enseigne le vaudou d'Afrique de l'Ouest [14] et celui de la Caraïbe [15] ? Que la mort n'est pas une fin et que, même, il n'y a pas de mort, mais une succession de vies d'aspects différents, une transmission d'énergie vitale d'un corps humain à la nature, puis à un autre corps humain.

Alors, définir la mort n'a peut-être pas de véritable sens, sauf pour la loi, qui a besoin de limites nettes pour la sauvegarde des intérêts des individus et de l'État. Mais à titre individuel, personnel, à quoi bon définir la mort?

Si, pour Xavier Bichat, «La vie est l'ensemble des fonctions qui résistent à la mort », définir la mort comme l'opposition à la vie n'a pas de sens. La mort s'oppose à la naissance, pas à la vie, car il n'y a pas de vie sans mort. La vraie mort, c'est la disparition, l'altération, l'éloignement. Dans beaucoup de sociétés lointaines, celui qui est mort, c'est celui dont on ne se souvient ni des traits du visage, ni du nom [16]. Celui-là est vraiment mort, sans l'ombre d'un doute. Mais celui qui, même en linceul dans un caveau (famadinha, à Madagascar) ${ }^{3}$, même à l'état de cendres dans une urne funéraire, même sous la forme d'une momie couverte d'or dans un temple (« bouddhas vivants » du Japon, de Taiwan ou de Thaïlande), même sous l'aspect d'un crâne surmodelé dans la case d'une société secrète (au Cameroun, Bénin, Nigéria ou en Papouasie-Nouvelle-Guinée), celui qui entretient encore un lien avec la communauté des vivants, celui qui intercède encore pour eux vis-à-vis des dieux ou des puissances surnaturelles, alors il n'est pas encore mort. $\diamond$

Death or deaths?

${ }^{2}$ Le Livre des morts tibétain présente les étapes de la préparation à la mort et donne une description du chemin menant vers l'Au-delà et de la vie Là-bas..

${ }^{3}$ Le famadihana, ou retournement (c'est-à-dire ré-inhumation) des morts, est une coutume funéraire que l'on rencontre dans certaines régions de Madagascar.
LIENS D'INTÉRÊT

L'auteur déclare n'avoir aucun lien d'intérêt concernant les données publiées dans cet article.

\section{RÉFÉRENCES}

1. Ad Hoc Committee of the Harvard medical school to examine the definition of brain death. a definition of irreversible coma: report of the ad hoc committee of the harvard medical school to examine the definition of brain death. JAMA $1968 ; 205: 337-40$

2. Charlier P, Hassin J. La mort sociale : réflexions éthique et d'anthropologie médicale. Ethics Med Public Health $2015 ; 1$ : 512-6.

3. Dreier JP, Fabricius M, Ayata C, et al. Recording, analysis, and interpretation of spreading depolarisations in neurointensive care: review and recommendations of the COSBID research group. J Cereb Blood Flow Metab $2017 ; 37: 1595-625$.

4. Kondziella D, Olsen MH, Lemale CL, Dreier JP. Migraine aura, a predictor of near-death experiences in a crowdsourced study. Peer J $2019 ; 7$ : e8202.

5. Charlier P, Bou Abdallah F, Deo S, Hassin J. Are Near-Death-Experience (NDE) only liberation of endogenous hallucinogens? Resuscitation 2018 ; 124 : ell-2.

6. Mashour GA, Frank L, Batthyany A, et al. Paradoxical lucidity: a potential paradigm shift for the neurobiology and treatment of severe dementias. Alzheimers Dement 2019; 15 : 1107-14.

7. Laureys S. Un si brillant cerveau. Les états limites de conscience. Paris : Odile Jacob, 2015.

8. Claassen J, Doyle K, Matory A, et al. Detection of brain activation in unresponsive patients with acute brain injury. N Engl J Med 2019 ; 380 : 2497-505.

9. Forti A, Brugnaro P, Rauch S, et al. Hypothermic cardiac arrest with full neurologic recovery after approximately nine hours of cardiopulmonary resuscitation: management and possible complications. Ann Emerg Med $2019 ; 73: 52-7$.

10. Ferreira PG, Munoz-Aguirre M, Reverter F, et al. The effects of death and post-mortem cold schema on human tissue transcriptomes. Nat Comm $2018 ; 9: 490$.

11. Pozhitkov AE, Neme R, Domazet-Lošo T, et al. Tracing the dynamics of gene transcripts after organismal death. Open Biol $2017 ; 7: 160267$.

12. Zhu $Y$, Wang $L$, Yin $Y$, Yang $\varepsilon$. Systematic analysis of gene expression patterns associated with postmortem interval in human tissues. Sci Rep $2017 ; 7$ : 5435.

13. Charlier P. Ouvrez quelques cadavres. Une anthropologie médicale du corps mort. Paris : Buchet-Chastel, 2015.

14. Charlier P. Vaudou. La nature, les dieux, les hommes (Bénin). Paris : Terre Humaine, 2020.

15. Charlier P. Zombis. Enquête sur les morts-vivants (Haïti). Paris : Tallandier, 2015.

16. Charlier P. Rituels. Paris : Le Cerf, 2020.

TIRÉS À PART

P. Charlier

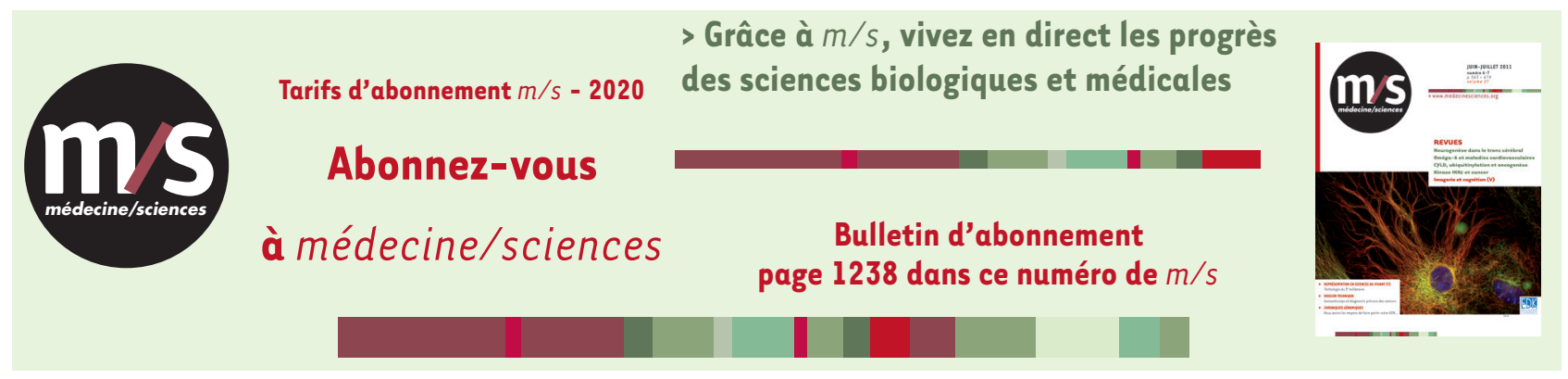

